Relations industrielles

Industrial Relations

\title{
Christophe DUPONT : La négociation. Conduite, théorie, application. Paris, Dalloz, 1982, 276 pp., ISBN 2-247-00315-X
}

\section{Paul Beaulieu}

Volume 40, numéro 4, 1985

URI : https://id.erudit.org/iderudit/051387ar

DOI : https://doi.org/10.7202/051387ar

Aller au sommaire du numéro

Éditeur(s)

Département des relations industrielles de l'Université Laval

ISSN

0034-379X (imprimé)

1703-8138 (numérique)

Découvrir la revue

Citer ce compte rendu

Beaulieu, P. (1985). Compte rendu de [Christophe DUPONT : La négociation.

Conduite, théorie, application. Paris, Dalloz, 1982, 276 pp., ISBN 2-247-00315-X].

Relations industrielles / Industrial Relations, 40(4), 892-893.

https://doi.org/10.7202/051387ar

Tous droits réservés @ C Département des relations industrielles de l'Université Laval, 1985
Ce document est protégé par la loi sur le droit d'auteur. L’utilisation des services d'Érudit (y compris la reproduction) est assujettie à sa politique d'utilisation que vous pouvez consulter en ligne.

https://apropos.erudit.org/fr/usagers/politique-dutilisation/ 
L'exploitation ingénieuse que l'auteur fait de ses sources d'inspiration théorique lui permet d'introduire une série de concepts nouveaux et stimulants pour l'analyse du déroulement du conflit de travail. Qu'il s'agisse du champ où se produit le conflit que l'auteur conceptualise comme un échiquier, des acteurs en interaction parmi lesquels il distingue les groupes opérants et les coalitions, et dont il analyse la capacité et le potentiel d'action, Gilles Plante introduit progressivement tout un outillage conceptuel permettant de mieux comprendre les aspects tactiques du conflit du travail. Il en est de même en ce qui concerne les aspects stratégiques du conflit où l'auteur va au-delà de la distinction classique entre l'offensive et la défensive pour en examiner les rapports mutuels et nous familiariser avec la réalité des manoeuvres stratégiques visant soit la conquête ou la défense de la liberté d'action, soit l'économie des forces.

Au total cependant, l'ouvrage de Gilles Plante apporte un éclairage nouveau davantage sur les aspects dynamiques du conflit du travail que sur la structure même du jeu qu'il constitue. À cet égard, l'auteur emprunte trop facilement la distinction issue de la théorie des jeux entre jeu à somme nulle et jeu à somme non nulle. Cette distinction ne me paraît guère prometteuse dans le cas des conflits du travail pour deux raisons. La première est que si l'on se place d'un point de vue objectif, extérieur aux acteurs eux-mêmes, l'immense majorité des conflits de travail appartient plutôt à la catégorie des jeux à somme non nulle, pour des raisons qui ont déjà été développées par Adam et Reynaud (Conflit du travail et changement social, P.U.F., 1978, pp. 131-138). Par contre, si l'on part de la subjectivité des acteurs sociaux, et qu'on privilégie ainsi une démarche d'intériorité, la plupart des conflits du travail apparaissent plutôt comme des jeux à somme nulle (chaque partie cherche à gagner en faisant perdre l'autre). En fait, la distinction entre ces deux structures de jeu n'est pas vraiment discriminante lorsqu'elle est transposée au domaine des relations du travail.

Enfin, il est dommage que l'auteur n'apporte aucune conclusion à son ouvrage. Pour ma part, je sors de la lecture de ce livre avec l'impression qu'il s'agit d'une oeuvre stimulante au plan intellectuel et qui, bien loin d'encourager à la multiplication des conflits du travail comme l'évoque Gérald Dion dans sa Préface, est plutôt de nature à inciter les parties à la prudence.

Marcel SIMARD

Université de Montréal

La négociation. Conduite, théorie, application par Christophe Dupont, Paris, Dalloz, 1982, 276 pp., ISBN 2-247-00315-X

Au cours des années soixante et soixante-dix, nous avons assisté à un développement impressionnant des connaissances sur le phénomène de la négociation. C'est dans le sillage de ce foisonnement de connaissances parcellaires qu'a débuté l'élaboration d'un champ d'étude nouveau à partir du domaine des sciences sociales. En ce milieu des années quatre-vingt, on ne peut affirmer qu'un corpus théorique unifié soit émergé de cet effort de construction sur l'objet. La négociation, en tant que champ disciplinaire de recherche, se situe au stade pré-paradigmatique (au sens de Kuhn). Les nombreux résultats de la recherche ont surtout permis la formulation de théories partielles sur le phénomène de la négociation mais qui font toutefois l'objet d'un certain consensus. Avec la décennie 80 , les recherches sur la négociation ont entamé une phase de bilan et de consolidation des connaissances disponibles; travail qui correspond, à un registre supérieur, aux efforts initiaux et très fructueux du début des années 60 par les tandems de Siegel-Fouraker, Sawyer-Guetzkow et Walton-McKersie. Cette tâche d'évaluation des connaissances sur la négociation collective constitue l'objectif que s'est fixé Christophe Dupont en écrivant cet ouvrage. 
Le texte est divisé en trois parties: les fondements de la conduite de la négociation, le fond et les modeles théoriques disponibles, et enfin les grands secteurs d'application des connaissances.

La première partie discute la spécificité de la négociation en introduisant le lecteur aux concepts d'acteurs, de divergence et d'arrangement dans le but de former une définition de l'activité pure de négociation. Viennent ensuite une série de considérations sur des dimensions clefs pour la conduite de la négociation: les enjeux, les mandats, la dynamique du pouvoir et les relations entre les acteurs. Toutes ces notions ont pour but d'en arriver par la suite à une description de la double nature de la négociation, ou pour être plus juste, du dilemme fondamental de cette activité sociale, c'est-à-dire les aspects intégratifs et distributifs de la négociation. Dupont a le mérite de très bien exposer l'instabilité et l'aspect multi-formes de cette double nature. Suivent les deux derniers chapitres de cette première partie qui sont consacrés à une synthèse, sobre dans son exposé mais complète en termes d'horizon, sur les stratégies de négociation, les tactiques et le développement de l'argumentation.

La seconde partie de l'ouvrage est littéralement un essai de synthèse des éléments théoriques disponibles sur la négociation. Dupont, après un bref regard sur les méthodologies de recherche utilisées à date pour l'étude de la négociation, fournit une typologie réussie de l'évolution des tendances de la recherche sur la négociation. Le lecteur qui recherche un point de vue d'ensemble sur la négociation y trouvera une «cartographie» adéquate. La période couverte est celle de 1955 à 1979. Quatre grandes catégories ont été retenues pour la classification des éléments théoriques: a) les théories psychologiques et psycho-sociologiques; b) la théorie économique et la théorie des jeux; c) les théories axées sur le processus; d) les recherches pragmatiques.

En ce qui concerne les modèles globaux, une attention particulière est portée au modèle causal de Sawyer et Guetzkon ainsi qu'à celui de Walton et McKersie. Au plan des modèles quantitatifs, Dupont porte un intérêt spécifique au modèle général de Bartos, car il s'agit d'un modèle global intégratif qui fusionne les «variables de déroulement» et les «(variables des stratégies et de résultats». Le modèle de Bartos est construit à partir d'un dispositif expérimental fonctionnant dans le cadre de la théorie des jeux.

Finalement, dans la dernière partie, l'auteur illustre trois des domaines particuliers d'application de la négociation, c'est-à-dire la négociation commerciale, la négociation des conditions de travail et la négociation internationale. Il s'agit de la partie de l'ouvrage qui n'offre pas le même niveau de profondeur que les précédents. L'auteur se limite principalement à un «exposé d'illustration» et circonscrit sur inventaire de la littérature en grande partie au contexte et aux sources françaises.

En somme, il s'agit d'une synthèse théorique très bien réussie, tant au niveau du contenu que de la forme, qui a le grand mérite de rendre disponible en langue française une intégration des connaissances générales disponibles sur le phénomène de la négociation formelle.

Paul BEAULIEU 